\title{
Cancers Primitifs Oto-Rhino-Laryngologiques Et Cervico-Maxillo-Faciaux De L'enfant: Aspects Épidémiologiques Et Histopathologiques
}

\author{
Foma W. \\ Amana B. \\ Pegbessou E. \\ Bissa $H$. \\ Adam S.
}

Service d'ORL et chirurgie cervico-maxillo-faciale

du CHU Sylvanus Olympio de Lomé

Dolou W.

Service d'ORL du CHU campus de Lomé

Darré T.

Laboratoire d'Anatomie Pathologique du CHU Sylvanus Olympio de Lomé.

\section{Kpemissi $\boldsymbol{E}$.}

Service d'ORL et chirurgie cervico-maxillo-faciale

du CHU Sylvanus Olympio de Lomé

doi: 10.19044/esj.2016.v12n24p389 URL:http://dx.doi.org/10.19044/esj.2016.v12n24p389

\begin{abstract}
Objective: Have a view on child's head and neck cancers in a reference centre in Togo.

Methodology: It was a descriptive retrospective study about the cancers diagnosed among children under 15 years in head and neck department of Sylvanus Olympio Teaching Hospital of Lomé in Togo from 1st January 2005 to 31 December 2014. The pieces were analysed in the pathological anatomy laboratory of the same teaching Hospital.

Results: The child's head and neck cancers represented $0.8 \%$ of the whole ENT tumours and $5.5 \%$ of head and neck cancers. The average age was 8 years \pm 4.7 ranging from 3 months to 15 years. The male sexe was predominant in 15 cases. The frequent location was ganglions, followed by oral cavity (gingivo-maxillary location and gingivo-mandibular location) in respectively 13 and 6 cases. The sinus, rhinopharynx, and larynx locations were found in 01 case of each cancer. In terms of histopathology, there were 21 cases of non-Hodgkin's lymphoma of which 09 cases of Burkitt's and 01 case of inferior lip neuroblastoma.
\end{abstract}


Conclusion: Child's head and neck cancers are scarce in Togo and dominated by malignant primitive cervical adenopathy.

Keywords: Child, Epidemiology, cancer, ENT

\section{Resume}

Objectif: Etablir le panorama des cancers oto-rhino-laryngologiques (ORL) et cervico-maxillo-faciaux (CMF) de l'enfant dans un service de référence au Togo.

Méthodologie: Il s'est agi d'une étude rétrospective descriptive portant sur les cancers diagnostiqués chez l'enfant de moins de 15 ans dans le service d'ORL et de chirurgie CMF du CHU Sylvanus Olympio de Lomé au Togo. Les pièces ont été analysées par le Laboratoire d'Anatomie Pathologique du même CHU.

Résultats : Les cancers ORL et CMF de l'enfant représentaient 0,8\% de l'ensemble des tumeurs ORL et 5,5\% des cancers ORL et CMF. L'âge moyen était de 8 ans $\pm 4,7$ avec des extrêmes de 3 mois et 15 ans. Le sexe masculin était prédominant avec 15 cas. La localisation la plus fréquente a été les ganglions, suivie de la cavité buccale (localisation gingivo-maxillaire et gingivo-mandibulaire) dans respectivement 13 et 06 cas. Les localisations sinusienne, rhinopharyngée et laryngée ont été retrouvées dans 01 cas chacune. Sur le plan histologique, nous avons retrouvé 21 cas de lymphome non hodgkinien dont 09 de type Burkitt et 01 cas de neuroblastome de la lèvre inférieure.

Conclusion: Les cancers ORL et CMF de l'enfant sont rares au Togo et sont dominés par les adénopathies cervicales malignes primitives.

Mots clés : Enfant, Epidémiologie, Cancer, ORL.

\section{Introduction}

Les cancers oto-rhino-laryngologiques (ORL) et cervico-maxillofaciaux (CMF) de l'enfant sont en général rares et ne sont dans la plupart des cas pas spécifiquement cervico-faciaux. Ils sont donc répertoriés le plus souvent selon l'histologie indépendamment du site (Patte, 2010). Au Togo, Amégbor, Darre, Padaro, Amewuame, Guédénon \& Napo-Koura (2011) rapportaient sur les cancers solides de l'enfant, une prédominance du lymphome non hodgkinien $(\mathrm{LNH})$ à localisation ganglionnaire et maxillaire. L'absence de données spécifiques sur la question dans notre pays qui de surcroit a une population très jeune (42\% ont moins de 15 ans) (Fonds des Nations Unies pour la Population [UNFPA], 2010) a motivé ce travail qui a pour but de dresser le panorama des cancers primitifs ORL et CMF de l'enfant dans un service de référence. 


\section{Materiels Et Methode}

Il s’est agi d'une étude rétrospective descriptive portant sur les cancers diagnostiqués chez l'enfant âgé d’au plus 15 ans dans le service d’ORL et de chirurgie cervico-maxillo-facial (CCMF) du CHU Sylvanus Olympio (S.O) de Lomé au Togo du 1er Janvier 2005 au 31 Décembre 2014 (10 ans). Notre matériel d'étude était constitué de dossiers des enfants et du registre de résultats d'examen histologique des pièces opératoires et des biopsies, du service d'ORL et de CCMF. Les pièces ont été analysées par le Laboratoire d'Anatomie Pathologique dudit CHU, utilisant comme techniques d'examen histologique, l'examen anatomopathologique conventionnel et les techniques histochimiques. Les variables étudiées étaient la fréquence, le sexe, l’âge, le motif de consultation, le siège du cancer ainsi que son type histologique.

\section{Resultats}

\section{Aspects épidémiologiques}

Nous avons colligé au cours de notre période d’étude 22 cas de cancers primitifs ORL et CMF de l'enfant représentant 0,8\% de l'ensemble des tumeurs ORL et 5,5\% des cancers ORL et CMF enregistrés durant la même période, soit une fréquence annuelle de 2,2 cas en moyenne. Le sexe masculin était atteint dans 15 cas et le sexe féminin dans 7 cas, soit une sexratio de 1,5. L'âge moyen était de 8 ans $\pm 4,7$ avec des extrêmes de 3 mois et 15 ans. Sept enfants avaient entre 0 et 5 ans, 7 autres entre 6 et 10 ans puis 8 entre 11 et 15 ans. Les motifs de consultation étaient la tuméfaction latérocervicale (13 cas) parfois ulcérée (Figure 1), la déformation faciale (6 cas) (Figure 2), la masse labiale inférieure (1 cas), l'obstruction nasale (1 cas) et la dyspnée laryngée (1 cas).

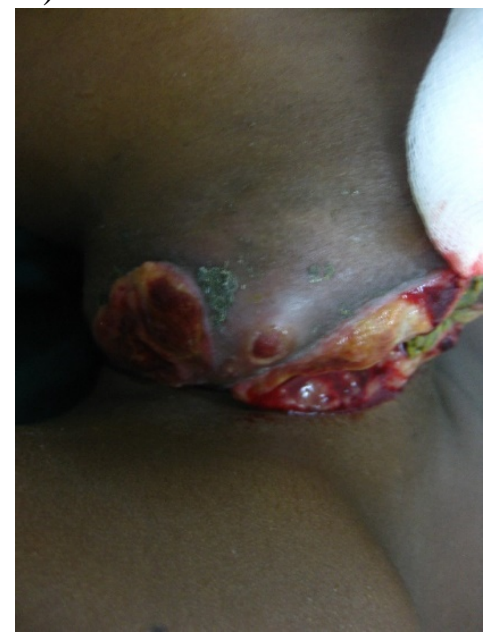

Figure 1 : Agglomérat d’adénopathies nécrotico-hémorragiques latéro-cervicales gauches révélant un lymphome non hodgkinien chez un garçon de 8 ans. 


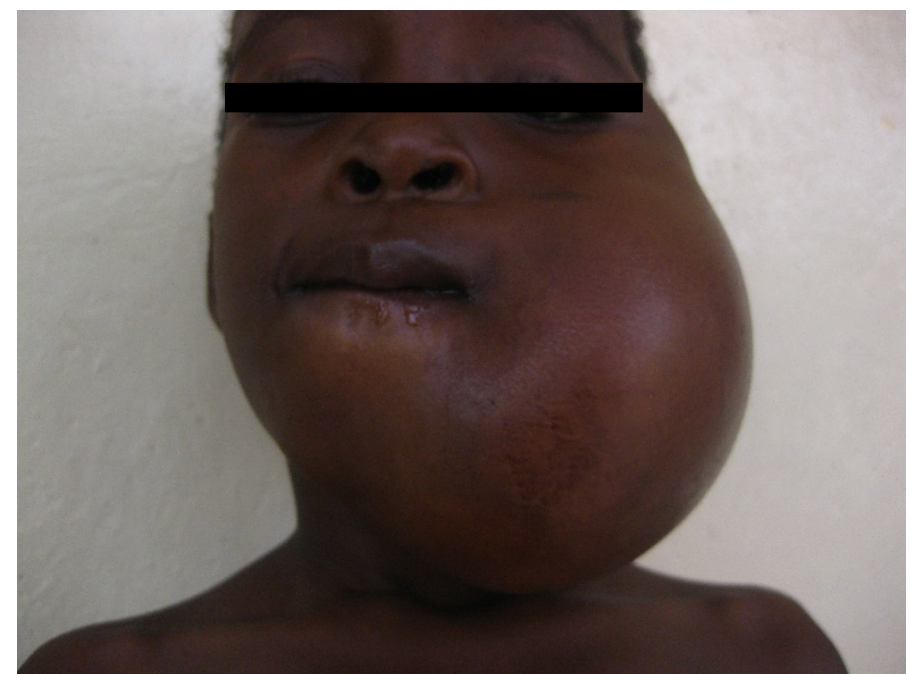

Figure 2 : Volumineuse masse gingivo-mandibulaire révélant un lymphome de Burkitt chez un garçon de 7 ans.

Tous les patients avaient consulté après une durée d'évolution d'au moins un mois. La localisation la plus fréquente a été les ganglions cervicaux, suivie de la cavité buccale (localisation gingivo-maxillaire et gingivo-mandibulaire) dans respectivement 13 et 06 cas. Les localisations sinusienne, rhinopharyngée et laryngée ont été retrouvées dans 01 cas chacune.

\section{Aspects histologiques}

Sur le plan histologique, nous avons retrouvé 21 cas de LNH et 01 cas de neuroblastome de la lèvre inférieure. Selon le type cellulaire de lymphome il s'agissait du lymphome de Burkitt (LBu) dans la majorité des cas (Tableau). Le LBu était de localisation buccale dans 5 cas, ganglionnaire dans 3 cas et sinusienne maxillaire dans 1 cas. Les cas de LNH ont été adressés au service d'oncologie pédiatrique du CHU S.O pour une chimiothérapie.

Tableau : Répartition des lymphomes non hodgkiniens selon le type cellulaire

\begin{tabular}{ccc}
\hline CIM-10 & Formulation internationale à usage clinique & Effectif \\
\hline C83-7 & J- Burkitt & 09 \\
C83-3 & G- Diffus à grandes cellules & 06 \\
C83-0 & A- Diffus à petits lymphocytes & 03 \\
C83-2 & F- Diffus mixte, à petites et grandes cellules & 02 \\
C83-1 & E- Diffus à petites cellules clivées & 01 \\
\hline & Total & $\mathbf{2 1}$
\end{tabular}




\section{Discussion}

Les cancers ORL et CMF de l'enfant ont représenté 5,5\% de l'ensemble des cancers du service durant la période d'étude. Plusieurs auteurs ont rapporté des fréquences supérieures à la nôtre : 11\% des moins de 15 ans au Nigeria (Otoh, Johnson, Danfillo, Adeleke \& Olasoji, 2004); 9,11\% des moins de 16 ans au Ghana (Abdulai, Nuamah \& Gyasi, 2012); 12\% des moins de 19 ans aux Etats-Unis (Albright, Topham \& Reilly, 2002). Ces observations montrent que les cancers ORL de l'enfant sont relativement rares au Togo. Cette fréquence relativement faible reflète celle de la série de Amegbor et al (2011), qui ont trouvé que les cancers solides de l'enfant de moins de 15 ans, représentaient $7 \%$ de l'ensemble des cancers au Togo. Tous ces auteurs ont rapporté une prédominance masculine. Le délai relativement long constaté avant la première consultation serait dû au manque d'information sur la nécessité de visites médicales et par certaines pratiques familiales ; les enfants ne sont amenés à l'hôpital qu'après l'échec du traitement traditionnel. Aussi, le personnel soignant vu en périphérie au début des symptômes est généralement peu qualifié pour la prise en charge de la pathologie ORL de l'enfant. Sur le plan histologique nous avons retrouvé 21 cas de LNH dont $09 \mathrm{LBu}$ et 01 cas de neuroblastome de la lèvre inférieure. En général, chez l'enfant, seuls quelques sous types de LNH sont observés : en majorité des LBu et des LNH lymphoblastiques et avec une moindre fréquence des LNH à grandes cellules (Patte, 2006). En effet les LNH sont l'un des cancers les plus fréquents de l'enfant africain et sont dominés par le $\mathrm{LBu}$. Sa localisation maxillo-faciale est caractéristique de l'enfant africain (Munck JN \& Schwaab G, 2001 ; Ségbéna , Kuéviakué, Vovor, Tatagan-agbi, Assimadi, Napo-koura \& Kpodzro, 1997 ; Stefan, 2015). Il y a déjà près de deux décennies, dans la série de Ségbéna et al (1997) dans un service de pédiatrie, le LBu avait représenté 73,68\% des LNH de l'enfant et $45,9 \%$ des cancers de l'enfant. La forte incidence du LBu observée dans certaines régions d'Afrique serait en rapport avec l'endémie du paludisme. L'explication en est l'association du paludisme aux infections virales telles celle du virus d'Epstein-Barr et du virus de l'immunodéficience humaine constituant un facteur de risque de survenue du LBu (Jaffe, Harris, Stein \& Vardiman, 2001 ; Kirk, Pedersen, Cozzi-Lepri, Antunes, Miller, Gatell...EuroSIDA Study Group, 2001).

\section{Conclusion}

Les cancers ORL et CMF de l'enfant sont rares au Togo, touchent le plus souvent les garçons et sont dominés par les adénopathies cervicales malignes primitives. Sur le plan histologique, il s'agissait de LNH dans la majorité des cas avec comme type cellulaire prédominant le LBu. 


\section{References:}

Abdulai AE, Nuamah IK, Gyasi R. (2012). Head and neck tumours in Ghanaian children. A 20 year review. International Journal of Oral \& Maxillofacial Surgery; 41(11):1378-82.

Albright JT, Topham AK, \& Reilly JS. (2002). Pediatric head and neck malignancies: US incidence and trends over 2 decades. Archives of Otolaryngology - Head \& Neck Surgery;128(6):655-9.

Amégbor K, Darre T, Padaro E, Amewuame E, Guédénon J, \& Napo-Koura G. (2011, Feb) Cancers solides de l'enfant au Togo: aspects épidémiologiques et histopathologiques à propos de 365 cas observés au CHU Tokoin de Lomé. African Journal of Cancer; 3(1) : 24-8.

Jaffe ES, Harris NL, Stein H, \& Vardiman JW. (Eds.). (2001). Pathology and Genetics of tumors of haematopoietic and lymphoid tissues: World Health Organization Classification of Tumours. IARC Press, Lyon, pp 1814.

Kirk O, Pedersen C, Cozzi-Lepri A, Antunes F, Miller V, Gatell JM...EuroSIDA Study Group. (2001). Non-Hodgkin lymphoma in HIVinfected patients in the era of highly active antiretroviral therapy. Blood, 98:3406-12.

Munck JN \& Schwaab G. (2001). Lymphomes des voies aérodigestives supérieures. Encycl Méd Chir (Elsevier SAS, Paris), Oto-rhino-laryngologie, 20-957-A-10, 6 p.

Otoh EC, Johnson NW, Danfillo IS, Adeleke OA \& Olasoji HA. (2004). Primary head and neck cancers in North Eastern Nigeria. West African Journal of Medicine ; 23 (4) : 305-13.

Patte C. (2006). Les lymphomes malins non-hodgkiniens de l'enfant. Oncologie; 8: 543-5.

Patte C. (2010). Tumeurs pédiatriques rares. In : Droz JP, Ray-Coquard I, Peix JL. Tumeurs malignes rares. Paris : Springer-Verlag France, p 379-81.

Ségbéna AY, Kuéviakué M, Vovor A, Tatagan-agbi K, Assimadi K, Napokoura G \& Kpodzro K. (1997). Le lymphome de Burkitt au Togo: aspects épidémiologique, clinique, thérapeutique et évolutif. Médecine d'Afrique Noire ; 44:141-6.

Stefan DC. (2015). Patterns of distribution of childhood cancer in Africa. J Trop Pediatr; 61(3):165-73.

UNFPA-Togo. (2012). Togo : 4ème recensement général de la population et de l'habitat 2010. consulté le 05/05/2016 à 22 heures, http://countryoffice.unfpa.org/togo/drive/BONNEPRATIQUERGPH_TOGO .pdf. 